International Journal of Biomedicine | June 2021 - Volume 11, Issue Suppl_1: Abstracts from the Third Russian International Conference "Cryo-electron microscopy 2021: achievements and prospects"

POSTER ABSTRACT PRESENTATIONS

SESSION TITLE: STRUCTURE AND FUNCTIONS OF THE TRANSCRIPTION AND TRANSLATION APPARATUS OF THE CELL

DOI: 10.21103/IJBM.11.Suppl_1.P21

\title{
Abstract P-21: The Investigation of S.aureus Ribosome-Binding Factor A Localization on the 30S Ribosomal Subunit by Cryo-Electron Microscopy
}

Aydar G. Bikmullin ${ }^{1}$, Artem Stetsenko ${ }^{2}$, Alexander Golubev ${ }^{1,3}$, Liliia Nurullina $^{3}$, Iskander Khusainov ${ }^{4}$, Evelina Klochkova ${ }^{1}$, Natalia Garaeva ${ }^{1}$, Konstantin Usachev ${ }^{1}$

${ }^{1}$ Kazan Federal University, Kazan, Russia

${ }^{2}$ University of Groningen, Groningen, the Netherlands

${ }^{3}$ Institut de Génétique et de Biologie Moléculaire et Cellulaire, Université de Strasbourg, Illkirch, France

${ }^{4}$ Department of Molecular Sociology, Max Planck Institute of Biophysics, Frankfurt am Main, Germany

Background: Ribosome biogenesis is a complex process of ribosomal RNA and protein binding. Bacterial ribosome maturation and components involved in it are especially interesting, because they are widespread targets for antibiotics. A number of special protein factors facilitating the maturation of the 30S small ribosomal subunit are known. One of them is a ribosome-binding factor A (RbfA). This is a small $(\sim 14 \mathrm{kDa})$ protein with $\mathrm{KH}$-domain organization distinguishing RNA binding proteins. Recent cryo-EM reconstruction of E.coli 30S-RbfA complex indicates that RbfA binds to $30 \mathrm{~S}$ subunit on the central decoding region and promotes the switch from the immature state of h28 (neck) to mature state. RbfA interacts with 3 -end of $16 \mathrm{~S}$ rRNA on mRNA exit channel and stabilizes the conformation of the region between $\mathrm{h} 28, \mathrm{~h} 44 / \mathrm{h} 45$ linker and $3 `$-end.

Methods: Pure S.aureus RbfA was obtained by homologous expression in E.coli BL21 strain followed by Ni-NTA and gel filtration. The $30 \mathrm{~S}$ subunits were obtained by dissociation of the S.aureus $70 \mathrm{~S}$ ribosomes in a sucrose gradient (0-30\%). We performed 30S subunit and RbfA complex reconstitution, sample and grid preparation. Data was collected on Talos Arctica, Falcon 2 detector (FEI Company/Thermo Fisher). 
Results: The 30S-RbfA complex density map with average resolution $\sim 3.5 \AA$ ( $F S C=0.143$ ) was obtained. In comparison with the free subunit map (EMD 23052) we observed an extra density on the neck region near the decoding center region.

Conclusion: Obtained data is correlated with recent structural results of the homologous E.coli RbfA. We consider that S.aureus RbfA binds to the $30 \mathrm{~S}$ subunit at the same region. The next step of our structural research is building the model of S.aureus 30S-RbfA complex.

Key Words: ribosome $\bullet$ 30S subunit biogenesis $・$ RbfA $\bullet$ protein translation

This work was supported by the Russian Foundation for Basic Research (Grant No. 20-54-15001)

*Corresponding author: Aydar G. Bikmullin. E-mail: aydar.bikmullin@gmail.com

International Journal of Biomedicine. 2021;11 Suppl 1: S20-21.

doi: 10.21103/IJBM.11.Suppl_1.P21

(C)2021 International Medical Research and Development Corporation 